\title{
High Variability in Radiologists' Reporting Practices for Incidental Thyroid Nodules Detected on CT and MRI
}

\author{
J.K. Hoang, A. Riofrio, M.R. Bashir, P.G. Kranz, and J.D. Eastwood
}

\begin{abstract}
BACKGROUND AND PURPOSE: There are no guidelines for reporting incidental thyroid nodules seen on CT and MR imaging. We evaluated radiologists' current reporting practices for incidental thyroid nodules detected on these imaging modalities.
\end{abstract}

MATERIALS AND METHODS: Radiologists were surveyed regarding their reporting practices by using 14 scenarios of incidental thyroid nodules differing in size, patient demographics, and clinical history. Scenarios were evaluated for the following: 1) radiologists' most commonly selected response, and 2) the proportion of radiologists selecting that response (degree of agreement). These measures were used to determine how the patient scenario and characteristics of the radiologists affected variability in practice.

RESULTS: One hundred fifty-three radiologists participated. In 8/14 scenarios, the most common response was to "recommend sonography." For the other scenarios, the most common response was to "report in only body of report." The overall mean agreement for the 14 scenarios was $53 \%$, and agreement ranged from $36 \%$ to $75 \%$. Smaller nodules had lower agreement: $43 \%-51 \%$ for 8-mm nodules compared with $64 \%-75 \%$ for 15 -mm nodules. Agreement was poorest for the 10 -mm nodule in a 60 -year-old woman (36\%) and for scenarios with additional history of lung cancer (39\%) and multiple nodules (36\%). There was no significant difference in reporting practices and agreement when radiologists were categorized by years of practice, practice type, and subspecialty $(P>.55)$.

CONCLUSIONS: The reporting practice for incidental thyroid nodules on CT or MR imaging is highly variable among radiologists, especially for patients with smaller nodules $(\leq 10 \mathrm{~mm})$ and patients with multiple nodules and a history of cancer. This variability highlights the need for practice guidelines.

ABBREVIATIONS: ASNR = American Society of Neuroradiology; ITN = incidental thyroid nodule

ncidental thyroid nodules are seen in $16 \%-18 \%$ of CT and MR imaging studies that include the thyroid., ${ }^{1,2}$ Although the prevalence of malignancy in incidental thyroid nodules (ITNs) is low and small thyroid cancers have an excellent prognosis, concern for missing malignancy may nevertheless lead to further evaluation for small nonspecific thyroid nodules. Initiating a work-up of an ITN seen on CT or MR imaging with diagnostic sonography can lead to further costly procedures, including fine-needle aspi-

Received August 23, 2013; accepted after revision September 25.

From the Departments of Radiology, Division of Neuroradiology (J.K.H., A.R., P.G.K. J.D.E.), Abdominal Imaging (M.R.B.), and Radiation Oncology (J.K.H.), Duke University Medical Center, Durham North Carolina.

Please address correspondence to Jenny K. Hoang, MD, Department of Radiology, Duke University Medical Center, Box 3808, Erwin Rd, Durham NC, 27710; e-mail: jennykh@gmail.com

三 Indicates article with supplemental on-line table.

http://dx.doi.org/10.3174/ajnr.A3834 ration, follow-up sonography examinations, or even diagnostic thyroid lobectomy.

The Society of Radiologists in Sonography and other societies have published recommendations for biopsy of nodules seen on sonography, ${ }^{3}$ but no medical organizations have specific published recommendations for the work-up of thyroid nodules seen on CT and MR imaging. ${ }^{4}$ The Society of Radiologists in Sonography recommendations cannot be simply extrapolated to CT- and MR imaging-detected nodules because the sonographic signs of microcalcifications and solid composition cannot be reliably appreciated on CT and MR imaging. ${ }^{5}$ Furthermore, CT and MR imaging allow a more comprehensive evaluation of neck nodes than is possible with the limited number of images captured during a thyroid sonography examination.

Without technique-specific guidelines, the reporting of ITNs seen on CT and MR imaging is likely to be nonuniform and influenced by radiologists' practice types or personal opinions. This variation leads to inconsistent practices and the potential for con- 
Table 1: The 14 survey scenarios ${ }^{\mathrm{a}}$

\begin{tabular}{l} 
Scenario \\
\hline 8-mm nodule \\
12-year-old girl \\
30-year-old woman \\
30-year-old man \\
60-year-old woman \\
10-mm nodule \\
12-year-old girl \\
30-year-old woman \\
30-year-old man \\
60-year-old woman \\
15-mm nodule \\
12-year-old girl \\
30-year-old woman \\
30-year-old man \\
60-year-old woman \\
8-mm nodule in 60-year-old woman with history of \\
treated lung cancer \\
10-mm nodule in 60-year-old woman with 7 thyroid \\
nodules \\
\hline a Radiologists were given an introduction that an indeterminate thyroid nodule was \\
incidentally seen on CT or MRI with the above descriptions of nodule size and patient \\
history.
\end{tabular}

fusion among clinicians who receive the radiology reports. In a retrospective study, Yousem et $\mathrm{al}^{2}$ found that $61 \%$ of ITNs seen on CT and MR imaging of the neck were not reported by the radiologist issuing the clinical report, and they proposed that either the nodule was not seen or it was regarded as unimportant. A prior survey on incidental findings queried radiologists about the ITN, but the survey was limited to 1 scenario and was sent only to academic body imaging radiologists. ${ }^{6}$

The aim of this study was to survey radiologists' self-described reporting practices of hypothetic scenarios of ITNs detected on CT and MR imaging. We hypothesized that reporting practices for ITNs are highly variable and may depend on the radiologist's experience, practice type, and training.

\section{MATERIALS AND METHODS}

This study was approved by our institutional review board. The need for written informed consent was waived due to the design of this survey study and the anonymity of the survey respondents.

\section{Survey and Study Group}

A survey was designed to query radiologists about their reporting practices for 14 scenarios of ITN, differing in nodule size, age and sex of the patient, multiplicity of nodules, and presence of a history of treated lung cancer. The scenarios are listed in Table 1. The survey scenarios were designed to simulate the spectrum of ITNs encountered in clinical practice and to cover categories in the Duke 3-tiered system, which was devised for guiding work-up for ITNs detected on CT and MR imaging. ${ }^{1,4}$ These criteria are divided into 3 mutually exclusive categories: Category 1 consists of nodules of any size with aggressive imaging findings, including associated suspicious lymph nodes, local invasion, or focal metabolic activity on PET. Nodules not meeting the criteria for category 1 are considered to have indeterminate imaging findings on CT and MR imaging and are grouped into categories 2 and 3. Category 2 nodules are those of any size in patients younger than 35 years of age, and category 3 is nodules $\geq 15 \mathrm{~mm}$ in patients 35 years of age or older. The 3 categories represent a descending risk of malignancy and need for work-up. ITNs that do not belong to any of the 3 categories are not selected for work-up. Younger patients are in category 2 because other studies have shown that there is a higher ratio of malignant-to-benign nodules in younger patients. ${ }^{5,7-11}$

Survey takers were given the following 5 response choices for each scenario: A) do not report, B) report in only body of report, C) report in impression without recommendation, D) recommend sonography \pm biopsy, and E) I have no consistent practice. The survey also obtained information regarding the radiologists, including years in practice, current practice type, and subspecialty training.

The survey was created by using the Web site http://www. surveymonkey.com and was sent electronically to 3 groups: 1) radiologists in all subspecialties at a tertiary care academic institution (Duke University Medical Center), 2) radiologists attending the American College of Radiology head and neck radiology course, and 3) members of the American Society of Neuroradiology (ASNR). The survey was sent via e-mail lists to the first 2 groups. For ASNR members, the survey was featured in one of the monthly ASNR on-line newsletters that was e-mailed to all ASNR members. Only responses from board-certified radiologists were included. Responses were collected between September 18, 2012, and December 11, 2012.

\section{Analysis of Survey Results}

The results were evaluated for the following: 1) the most commonly selected response (A-E) for the 14 ITN scenarios, and 2) the degree of agreement for the most commonly selected response. The degree of agreement was the proportion of respondents who chose the most common response. A higher proportion would indicate high agreement and little variability in practice. The degree of agreement was categorized into high $(\geq 75 \%)$, moderate $(50 \%-74 \%)$, and low $(<50 \%)$.

\section{Response and Agreement by Scenario}

The response and degree of agreement for all respondents were evaluated to determine how nodule size, patient demographics, multiplicity of nodules, and cancer history would affect the reporting practice and variability in practice.

\section{Response and Agreement by Radiologist Groups}

Response and degree of agreement were compared for radiologists with different years in practice, practice type, and subspecialty training to determine whether these characteristics would affect the reporting practice and variability in practice.

The mean agreement was calculated for radiologists of each group. The paired $t$ test was used to compare differences in agreement between 2 groups of practices (academic versus private/hybrid). One-way ANOVA was used to compare differences in agreement for years in practice (fellow, attending of $<10$ years, and attending of $>10$ years) and differences in agreement in subspecialty training (neuroradiology, body imaging, and other).

Results from the on-line survey were exported on a spreadsheet for analysis (Excel; Microsoft, Redmond, Washington). Sta- 
Table 2: Characteristics of survey respondents

\begin{tabular}{lc}
\hline Characteristics of Groups & No. of Respondents \\
\hline Total & 153 \\
Years in practice & $27(18 \%)$ \\
Fellow & $76(50 \%)$ \\
Attending $<10$ years & $50(33 \%)$ \\
Attending $\geq 10$ years & \\
Practice type & $108(71 \%)$ \\
Academic & $45(29 \%)$ \\
Private or hybrid & \\
Subspecialty training & $100(65 \%)$ \\
Neuroradiology & $17(11 \%)$ \\
Abdominal radiology & $36(24 \%)$ \\
Combinations or other & \\
Survey group & $41(27 \%)$ \\
Duke Radiology & $28(18 \%)$ \\
ACR CME course & $84(56 \%)$ \\
ASNR members &
\end{tabular}

Note:-ACR CME indicates the American College of Radiology Continuing Medical Education.

tistical analyses were performed by using SAS Enterprise (Version 4.2; SAS Institute, Cary, North Carolina).

\section{RESULTS \\ Study Group}

One hundred fifty-three radiologists participated in the study, including 41 from Duke Radiology, 28 from the American College of Radiology course, and 84 ASNR members. The response rates for these 3 groups were $41 / 67$ (61\%), 28/36 (78\%), and 84/4552 (2\%), respectively. Table 2 shows the characteristics of the respondents. Seventy-one percent of radiologists were in academic practice, and $65 \%$ had received subspecialty neuroradiology training.

\section{Survey Results}

The On-line Table shows the most commonly selected response for each scenario and the degree of agreement for the most commonly selected response.

\section{Response and Agreement by Scenario}

Although there were 5 reporting options, there were only 2 answers that were commonly selected responses when all radiologists were grouped together: "report only in body" (B) or "recommend sonography" (D). "Recommend sonography" was the most common response for 8/14 scenarios: all 4 scenarios of $15-\mathrm{mm}$ nodules, 3 scenarios of $10-\mathrm{mm}$ nodules in patients 12 years and 30 years of age, and 1 scenario of an 8 -mm nodule in patients 12 years of age.

The degree of agreement represents the proportion of radiologists who chose the most commonly selected response and is an indicator of the most common practice. Agreement ranged from $36 \%$ to $75 \%$ (Fig 1), and the mean was 53\%. This finding indicates that only $53 \%$ of radiologists had common reporting practices.

Agreement for the most commonly selected response was influenced by nodule size. There was lower agreement for smaller nodules, with $43 \%-51 \%$ agreement for 8 - $\mathrm{mm}$ nodule scenarios compared with $64 \%-75 \%$ agreement for $15-\mathrm{mm}$ nodule scenarios. When 15-mm nodule scenarios were excluded, the mean agreement was $46 \%$ and the range was 36\%-56\%.
Agreement was similar for scenarios with different ages except for the 10 -mm nodule in the 60 -year-old patient, which had $36 \%$ agreement compared with $53 \%-56 \%$ for the same size nodule in younger patients. The sex of the patient in the scenario did not influence the response or agreement as evidenced by the scenarios with the male and female 30-year-old patients. The 2 special scenarios of a patient with an additional history of lung cancer and a patient with multiple nodules had poor agreement $39 \%$ and $36 \%$, respectively).

\section{Response and Agreement by Radiologist Subgroups}

The On-line Table shows that when comparing the 3 different groups of years in practice, there were 3 scenarios that had differences in the most commonly selected responses among the groups: 8 -mm nodules in 30-year-old men, $10-\mathrm{mm}$ nodules in 60 -year-old women, and multiple nodules (footnote $a$ on On-line Table). The mean agreement for fellows, attendings of $<10$ years, and attendings of $\geq 10$ years was $52 \%, 51 \%$, and $54 \%$, respectively, and it was not significantly different $(P=.81)$.

When practice type was considered, the most commonly selected responses differed between academic and private/hybrid practice radiologists for 2 scenarios in which the private/hybrid radiologists were more conservative, choosing "recommend sonography" and "report in impression without recommendation" rather than "report only in body" (On-line Table). These were 10 -mm nodules in a 60-year-old woman and a patient with multiple nodules, respectively. The mean agreement for academic and private/hybrid for both was $52 \%$, and it was not significantly different with the paired $t$ test $(P=.55)$.

The type of subspecialty training led to differences in the reporting for 4 scenarios. Radiologists with "other" training had "recommend sonography" rather than "report only in body" as the most commonly selected response for 8-mm nodules in male and female patients 30 years of age. Body-trained radiologists differed from the other subspecialties by choosing "report only in body" rather than "recommend sonography" for 10-mm nodules in 60-year-old women and patients with multiple nodules. The mean agreement for neuroradiology, body imaging, and other training was $52 \%, 54 \%$, and $53 \%$, respectively, and it was not significantly different $(P=.91)$.

\section{DISCUSSION}

In contrast to thyroid nodules detected on sonography, there are presently no specific recommendations or guidelines offered by professional radiology organizations that deal specifically with the problem of ITNs discovered on CT or MR imaging. ${ }^{3,4}$ This factor may contribute to confusion, inconsistent reporting, and even overinvestigation of small thyroid nodules. This study evaluates how radiologists claim to report thyroid lesions incidentally detected on CT and MR imaging and quantifies the variability in this self-described practice. We found that with the exception of relatively large nodules of $15 \mathrm{~mm}$, there is substantial variability in reporting practices, with $<50 \%$ of radiologists having the same practice of reporting ITNs. Additionally, variability is high even within groups similar in practice experience, practice type, and subspecialty.

Self-described reporting practices regarding incidental thy- 


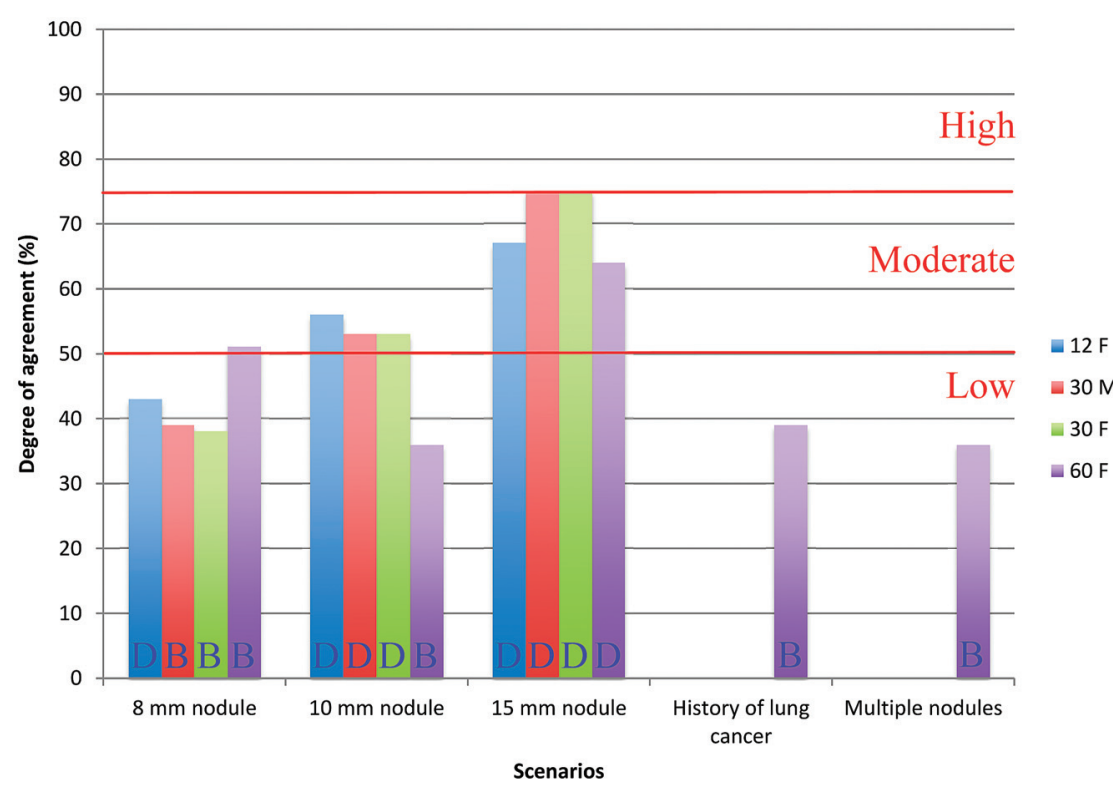

FIG 1. Degree of agreement for the most commonly selected response for all radiologists. Responses were the following: $\mathrm{B}=$ report only in body and $\mathrm{D}=$ recommend sonography. The degree of agreement was categorized into high ( $\geq 75 \%)$, moderate $(50 \%-74 \%)$, and low $(<50 \%)$. Scenarios included $12 \mathrm{~F}=12$-year-old girl, $30 \mathrm{M}=30$-year-old man, 30F $=30$-year-old woman, and $60 \mathrm{~F}=60$-year-old woman

roid nodules were previously investigated by Johnson et $\mathrm{al}^{6}{ }^{6}$ who included a scenario of a 1 -cm ITN in a 45 -year-old woman in a survey, along with other incidental-finding scenarios seen on body CT. They concluded that there was good agreement in reporting practices for ITNs, with $81 \%$ of radiologists recommending sonography in that scenario. For similar scenarios in our study, notably of 10 -mm nodules in a 30-year-old and a 60 -yearold woman, the most common responses by radiologists were "recommend sonography" for the younger woman but "report only in body" for the older woman. Our study also found much greater variability in reporting practices as demonstrated by a lower degree of agreement for these responses (53\% and 36\%, respectively). The differences between our results and those of Johnson et al could be attributable to our larger sample size (153 versus 27) and a more heterogeneous group of radiologists. In their work, Johnson et al specifically surveyed academic radiologists who had prior fellowship training in body imaging, while our study surveyed radiologists from a variety of practice types and subspecialties. We believe that better representation of all types of radiologists is one of the strengths of our study.

The scenarios with the greatest agreement of reporting practices were 15-mm nodules, for which the most common reporting option was to recommend sonography, regardless of age or sex. In contrast, the agreement was as low as $36 \%$ for a 60 -year-old woman with a 10 -mm nodule and a $10-\mathrm{mm}$ nodule in a patient with multiple thyroid nodules. An additional history of lung cancer in a 60 -year-old woman with an 8 -mm nodule also led to lower agreement (39\%) than when there was no lung cancer history (51\%). Low agreement in our survey reflects a small proportion of radiologists practicing in the same way. These inconsistent reporting practices could potentially result in confusion for the referring clinician and highlight the need for evidence-based or expert-opinion practice guidelines. Such guidelines should have specific recommendations for patient age, nodule size, and the number of nodules.

A review by Hoang et $\mathrm{al}^{4}$ proposed a set of criteria for reporting ITNs on CT, MR imaging, and PET. The work-up system, known as the Duke 3-tiered system, has since been evaluated in a retrospective study that compared the 3-tiered system with a $\geq 10$-mm-sized threshold for consecutive ITNs detected on CT neck studies. ${ }^{1}$ When the investigators applied the 3-tiered system to nodules of $\geq 10 \mathrm{~mm}$, they found that the work-up of nodules could be reduced by $46 \%$. In another study by Hobbs et al, ${ }^{12}$ the Duke 3-tiered system was applied to a cohort of ITNs undergoing fine-needle aspiration and found that the system would have prevented biopsy in 35\% of patients without missing any malignant nodules. Thus, guidelines such as the Duke 3-tiered system could reduce unnecessary ITN workups, improve consistency in reporting ITNs, and reduce the cost of medical care in this population.

When one is comparing our survey results with the results in the Duke 3-tiered system, it appears that the most commonly selected responses for scenarios by age and nodule size actually match the recommendations of the 3-tiered system. The exception is the 8 -mm nodules in the 30 -year-old patients, for which the survey respondents chose "report only in body," whereas the 3-tiered system does not specify a nodule size cutoff for the younger than 35-year age group. In clinical practice, the authors of the 3-tiered system use a 10-mm cutoff in adults younger than 35 years and no cutoff in children. These specifications were not described and used in the publications to simplify the guidelines for readers. Overall, the implication of the survey results is that the 3 -tiered system is concordant with current practices and could be implemented in clinical practice.

This study had several limitations. Although we attempted to survey a diverse group of radiologists, these results may not be generalizable to all radiologists because of the predominance of academic radiologists and neuroradiologists in our study population. Second, the response from the ASNR members was low at $2 \%$, though this is not surprising given that the survey was sent only once in an e-mailed newsletter. The absolute number of respondents from the ASNR was still large (86 radiologists), however, composing $56 \%$ of our study group. The response rate was higher from the other 2 groups of radiologists that received the survey. We also recognize that some radiology practices may have already developed guidelines within their groups for reporting thyroid nodules and that these groups may have already established high within-group agreement. In addition, there may be a nonresponse bias from radiologists who did not participate in the survey, which is inherent in survey studies. However, we do not believe that this would add systematic bias in favor of a particular 
result. Finally, these are self-described reporting practices and may not reflect the true practice of the reporting radiologists.

\section{CONCLUSIONS}

The reporting practice of ITNs on CT or MR imaging is highly variable among radiologists, especially for patients with smaller nodules ( $\leq 10 \mathrm{~mm}$ ) and for patients with multiple nodules and an additional history of cancer. This variability highlights the reality that there is no standard of practice and that there is a need for evidence-based or expert-opinion practice guidelines. Such guidelines could lead to greater reporting consistency and, potentially, decreased cost and medicolegal risk in the evaluation of low-risk ITNs.

Disclosures: Mustafa R. Bashir-UNRELATED: Consultancy: Bayer Healthcare, Siemens, Comments: honoraria for advisory board attendance, Grants/Grants Pending: Siemens. * Peter G. Kranz—UNRELATED: Other: Cephalogics LLC, * Comments: industry-sponsored investigation. ${ }^{*}$ Money paid to the institution.

\section{REFERENCES}

1. Nguyen XV, Choudhury KR, Eastwood JD, et al. Incidental thyroid nodules on CT: evaluation of 2 risk-categorization methods for work-up of nodules. AJNR Am J Neuroradiol 2013;34:1812-17

2. Yousem DM, Huang T, Loevner LA, et al. Clinical and economic impact of incidental thyroid lesions found with CT and MR. AJNR Am J Neuroradiol 1997; 18:1423-28

3. Frates MC, Benson CB, Charboneau JW, et al. Management of thy- roid nodules detected at US: Society of Radiologists in Ultrasound Consensus Conference Statement. Radiology 2005;237:794-800

4. Hoang JK, Raduazo P, Yousem DM, et al. What to do with incidental thyroid nodules on imaging? An approach for the radiologist. Semin Ultrasound CT MR 2012;33:150-57

5. Shetty SK, Maher MM, Hahn PF, et al. Significance of incidental thyroid lesions detected on CT: correlation among CT, sonography, and pathology. AJR Am J Roentgenol 2006;187:1349-56

6. Johnson PT, Horton KM, Megibow AJ, et al. Common incidental findings on MDCT: survey of radiologist recommendations for patient management. J Am Coll Radiol 2011;8:762-67

7. Belfiore A, La Rosa GL, La Porta GA, et al. Cancer risk in patients with cold thyroid nodules: relevance of iodine intake, sex, age, and multinodularity. Am J Med 1992;93:363-69

8. Fowler CL, Pokorny WJ, Harberg FJ. Thyroid nodules in children: current profile of a changing disease. South Med J 1989;82:1472-78

9. Kirkland RT, Kirkland JL, Rosenberg HS, et al. Solitary thyroid nodules in $\mathbf{3 0}$ children and report of a child with a thyroid abscess. Pediatrics 1973;51:85-90

10. Machens A, Holzhausen HJ, Dralle H. The prognostic value of primary tumor size in papillary and follicular thyroid carcinoma. Cancer 2005; 103:2269-73

11. Rago T, Fiore E, Scutari M, et al. Male sex, single nodularity, and young age are associated with the risk of finding a papillary thyroid cancer on fine-needle aspiration cytology in a large series of patients with nodular thyroid disease. Eur J Endocrinol 2010; 162:763-70

12. Hobbs H, Bahl M, Nelson RC, et al. Incidental thyroid nodules detected on imaging: can workup be reduced by using the Society of Radiologists in Ultrasound recommendations and the three-tiered system? AJR Am J Roentgenol 2014;202:18-24 